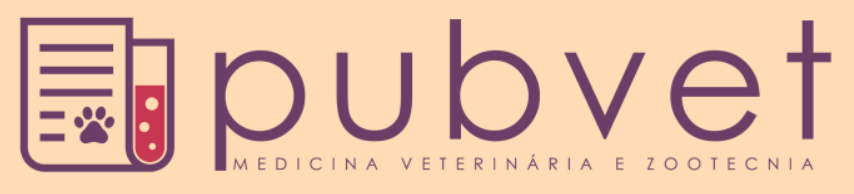

https://doi.org/10.31533/pubvet.v12n7a135.1-6

\title{
Abordagem fisioterapêutica em cão portador da Síndrome de Wobbler: Relato de caso
}

\author{
Maria Júlia Carrazzone Gouveia Padilha ${ }^{1}$, Lourival Barros de Sousa Brito Pereira ${ }^{1}$, Moisés \\ Bonifácio Alves Ferreira Filho ${ }^{1}{ }^{\bullet}$, Lucilo Bioni da Fonseca Filho ${ }^{1}{ }^{\bullet}$, Andréa Jullyanna de \\ Carvalho $^{1}$, Melissa Barbosa Pontes ${ }^{2}$, Nicolli de Albuquerque Leal Gomes D’Alcantara ${ }^{30}$, \\ Jordy Diniz de Oliveira Lima $^{3}$, Júlio Cézar dos Santos Nascimento ${ }^{4}$, Wagner McKlayton \\ Alves de Souza $^{4} \theta$ \\ ${ }^{I}$ Médico veterinário. Recife-PE Brasil. \\ ${ }^{2}$ Discente do curso de Medicina Veterinária em Centro Universitário Brasileiro. Recife-PE Brasil. \\ ${ }^{3}$ Discente do curso de Medicina Veterinária em Centro Universitário Mauricio de Nassau. Recife-PE Brasil. \\ ${ }^{4}$ Professorem Centro UniversitárioMauriciode Nassau, área de Medicina Veterinária.Recife-PEBrasil.E-mail:juliozootecnista@hotmail.com; isabelwagner@uol.com.br \\ *Autor para correspondência, E-mail: lorinho2013.1@hotmail.com
}

\begin{abstract}
RESUMO. A fisioterapia veterinária é uma área em expansão, que vem trazendo grande ganho à veterinária por proporcionar desde o simples alívio da dor à recuperação dos movimentos e melhora da qualidade de vida do animal. Define-se como Síndrome de Wobbler, uma pequena compressão no canal medular que pode ser causada por uma combinação de uma hérnia de disco ou um pequeno estreitamento do canal espinhal secundário a alterações ósseas que incidem sobre a medula espinhal. Afeta principalmente cães de raças grandes e gigantes, causando muitas dores e desconforto ao paciente. Apesar de ser uma afecção de indicação cirúrgica, a fisioterapia pode auxiliar no alivio das dores do paciente. Com este trabalho objetivou-se relatar um caso de uma cadela da raça dálmata de 11 anos, com mobilidade reduzida e sintomatologia dolorosa na região cervical, sendo esses sinais compatíveis com a Síndrome de Wobbler. O planejamento terapêutico incluiu exercícios de mobilização passiva de todas as articulações e alongamento da musculatura envolvida para evitar o agravamento das lesões. Estes exercícios também são indicados no tratamento da dor e na perda de mobilidade secundaria à disfunção neuromúsculoesquelética. No alivio da dor também é utilizado o TENS (Transcutaneous Electrical Nerve Stimulation) que é uma corrente analgésica, que atua nos nervos periféricos e sistemas modulares da dor, causando analgesia. A Bola Suíça foi utilizada neste caso com fins de alongamento, fortalecimento muscular, reeducação da postura e treino de equilíbrio. Apesar do animal ter sido encaminhado para o neurologista para reavaliação e possível encaminhamento cirúrgico, a melhora no quadro clínico de dor, da mobilidade dos membros, amplitude de movimento e melhora nas atividades cotidianas do animal foram eficientes principalmente pelas observações da tutora.
\end{abstract}

Palavras chave: alívio da dor, alongamento, reabilitação

\section{Physiotherapeutic approach in a dog with Wobbler's Syndrome: Case report}

ABSTRACT. Veterinary physiotherapy is an expanding area, bringing large gains to vet, by providing from simple pain relief to the recovery of the movements and improves the quality of life of the animal. Defined as Wobbler's syndrome, a little compression on the spinal canal that can be caused by a combination of a herniated disc or a small narrowing of the spinal canal secondary to bone alterations that affect the spinal cord. Mainly affects 
large breed and giant dogs, causing a lot of pain and discomfort to the patient. Despite being a pathology of surgical, physical therapy can aid in the relief of the patient's pain. With this work the aim report a case of a dog breed Dalmatian of 11 years, with reduced mobility and painful symptoms in the cervical region, being these signals compatible with the Wobbler syndrome. The therapeutic planning included passive mobilization exercises all the joints and stretching of the muscles involved to prevent further injuries. These exercises are also indicated in the treatment of pain and loss of mobility secondary to neuromuscle-skeletal dysfunction. The pain relief is also used the TENS (Transcutaneous Electrical Nerve Stimulation), which is an analgesic current, which acts on peripheral nerves and modular systems of pain, causing analgesia. The ball was used in this case Switzerland aimed at stretching, strengthening, muscular re-education of posture and balance training. Although the animal has been forwarded to the neurologist for reevaluation and possible surgical referral, an improvement in clinical of pain, mobility, and improves range of motion in the activities of the day of the animal the owner shows efficient comments.

Keywords: painfull, stretching, rehabilitation

\section{Enfoque fisioterapéutico en perro portador del Síndrome de Wobbler: Relato de caso}

RESUMEN. La Fisioterapia Veterinaria es un área en expansión, que viene trayendo grandes ganancias a la Veterinaria, por proporcionar desde el simple alivio del dolor a la recuperación de los movimientos y mejora de la calidad de vida del animal. Se define como Síndrome de Wobbler, una pequeña compresión en el canal medular que puede ser causada por una combinación de una hernia de disco o un pequeño estrechamiento del canal espinal secundario a alteraciones óseas que inciden sobre la médula espinal. Afecta principalmente a perros de razas grandes y gigantes, causando mucho dolor y molestias al paciente. A pesar de ser una afección de indicación quirúrgica, la fisioterapia puede auxiliar en el alivio de de dolor del paciente. Con este trabajo se objetivó relatar un caso de una perra de la raza dálmata de 11 años, con movilidad reducida y sintomatología dolorosa en la región cervical, siendo estas señales compatibles con el Síndrome de Wobbler. La planificación terapéutica incluyó ejercicios de movilización pasiva de todas las articulaciones y estiramiento de la musculatura involucrada para evitar el agravamiento de las lesiones. Estos ejercicios también se indican en el tratamiento del dolor y la pérdida de movilidad secundaria a la disfunción neuromuscular-esquelética. En el alivio del dolor también se utiliza el TENS (Transcutánea Electrical Nerve Stimulation), que es una corriente analgésica, que actúa en los nervios periféricos y sistemas modulares del dolor, causando analgesia. La Bola Suiza fue utilizada en este caso con fines de estiramiento, fortalecimiento muscular, reeducación de la postura y entrenamiento de equilibrio. A pesar de que el animal fue encaminado al neurólogo para reevaluación y posible encaminamiento quirúrgico, la mejora en el cuadro clínico de dolor, de la movilidad de los miembros, amplitud de movimiento y mejora en las actividades cotidianas del animal fueron eficientes principalmente por las observaciones de la tutora.

Palabras clave: alivio del dolor, estiramiento, rehabilitación

\section{Introdução}

A fisioterapia animal é uma área que estuda, previne e trata distúrbios cinéticos funcionais gerados por alterações genéticas, traumas ou doenças adquiridas, tendo bases científicas estabelecidas para seres humanos e animais ( $\underline{\text { Steiss }}$ \& Levine 2005). Cada vez mais os médicos veterinários que atuam na ortopedia estão vendo a necessidade de incorporar a especialidade no tratamento para melhorar a recuperação e a qualidade de vida dos animais (Levine et al. 2008).

A Síndrome de Wobbler, também conhecida como Espondilomielopatia Cervical (EMC) é uma patologia da região da coluna cervical, mais comumente encontrada em cães da raça Dobermann; porém podendo também afetar outras 
raças grandes e gigantes. Todos os cães com CSM (Cervical Spondylomyelopathy) têm algum grau de estenose do canal vertebral (Costa 2010). Esta estenose relativa do canal vertebral não condiz aos sinais clínicos, mas predispõe-se ao desenvolvimento de sinais neurológicos e motores (Costa 2010). Segundo Costa (2010), apesar das causas serem desconhecidas, existem estudos que tentam comprovar que a síndrome pode ser de origem genética, congênita, pela conformação do corpo do cão e/ou nutricional; porém existe pouca literatura que comprove tais causas.

O diagnóstico é feito inicialmente pelos sinais clínicos do paciente. Inicialmente aparecendo nos membros pélvicos (MMPP) e, posteriormente, nos torácicos (MMTT), podendo até passar despercebido nos MMTT. Segundo Costa (2010) a marcha dos membros torácicos pode aparecer de forma curta ou espástica. Esta pseudo-hipermetria é o resultado da liberação do neurônio motor superior causando rigidez/espasticidade, e difere da hipermetria verdadeira, pelo que os membros mostram o "alto passo" secundário à hiperflexão das articulações do membro torácico. Os déficits de reação postural (déficits de posicionamento proprioceptivo) são observados na maioria dos cães com EMC, mas podem não ser evidentes naqueles com história crônica apesar da presença de ataxia proprioceptiva (Costa 2010). A radiografia não pode dar o diagnóstico definitivo da EMC, mas é usada para descartar outras patologias como neoplasia óssea, traumatismo e osteomielite vertebral. As características radiográficas em cães de raça gigante com compressões ósseas podem ser vistas em projeções laterais e ventrodorsais. O diagnóstico também pode ser realizado a partir da mielografia, que segundo Costa (2010) um recente estudo comparando a mielografia com a ressonância magnética (RM) mostrou que a mielografia pode indicar uma localização errônea da principal lesão compressiva ou minimizar a severidade das lesões presentes. A tomografia computadorizada (TC) também auxilia no diagnostico final da Síndrome de Wobbler, principalmente quando combinada com a mielografia (Costa 2010).

O tratamento conservativo (medicamentoso) da Síndrome de Wobbler é temporário, usado somente para alivio dos sinais clínicos do paciente, usando corticosteroides como a dexametesona $(0.25 \mathrm{mg} / \mathrm{kg}$ a cada $24 \mathrm{~h})$, melhoram a função neurológica na compressão crônica da medula espinhal predominantemente por diminuição do edema vasogênico; e antiinflamatórios como prednisona na dosagem de 0,5 a $1,0 \mathrm{mg} / \mathrm{kg}$ a cada $12 \mathrm{~h}$ ou $24 \mathrm{~h}$. Segundo Costa (2010), a decisão de recomendar o tratamento cirúrgico deve basear-se em fatores, como a gravidade dos sinais neurológicos, o grau de dor, tipo de gravidade das lesões compressivas, resposta ou falta dela ao gerenciamento médico de cooperações de curto e longo prazo do proprietário e outros problemas neurológicos ou ortopédicos concorrentes ou doenças extraneurológicas. A fisioterapia também foi relatada no tratamento de cães com mielopatias cervicais graves e também pode ser usada no tratamento de cães com EMC auxiliando no alivio de dores cervicais, musculares e articulares do paciente e também prevenindo possíveis dores musculares e articulares por mau uso e/ou desuso dos membros devido as dores ( $\underline{\text { Costa 2010)}}$.

O prognóstico é extremamente variável, pois depende do nível de dor, da evolução da doença e do tratamento a ser usado no paciente, porém nos casos de Wobbler, dependendo do grau de dor do animal, a eutanásia deve ser considerada. O presente trabalho tem como objetivo relatar o caso de uma cadela da raça dálmata com 11 anos, diagnosticada com síndrome de Wobbler e submetida a fisioterapia.

\section{Relato de caso}

Foi atendido em uma Clínica Veterinária situada na região metropolitana do Recife, um canino, fêmea, da raça Dálmata, com 11 anos de idade e $20 \mathrm{~kg}$. Foi relatado pela tutora que a cadela há mais ou menos um ano e meio, apresentou dificuldades em deitar e se levantar, perde o equilíbrio ao fazer suas necessidades fisiológicas, caindo em cima da sua urina e fezes; tem dificuldades em se alimentar com a cabeça baixa e deambula com dificuldade. $\mathrm{O}$ animal foi encaminhado para uma radiografia cervical. Baseado nos achados, a paciente foi conduzida para fisioterapia para possível melhora do quadro clínico, com foco no alívio e prevenção das dores, fortalecimento muscular e reestabelecer seu equilíbrio.

$\mathrm{Na}$ avaliação, constatou-se a presença de andar rígido, quedas e cifose, não aparentando dor cervical, porém demonstrando desconforto quando realizado movimentos de flexão da região cervical. Constatou-se, também, que os reflexos dos membros anteriores e posteriores se apresentavam aumentados, sensibilidades para dor superficial e profunda preservadas, micção e 
defecação preservadas, ausência de alterações em nervos cranianos, configurando-se, assim, uma síndrome cervical. Também foi observado na avaliação, que a pupila do animal se apresentava dilatada e a tutora relatou que permanecia assim em casa, o que pode ser um indicativo de dor.

$\mathrm{Na}$ radiografia simples foi encontrada diminuição de espaço intervertebral (Figuras $1 \mathrm{e}$ 2) entre C5-C6 e C6-C7, que, juntamente com a clínica apresentada, caracteriza uma Síndrome de Wobbler onde os achados radiográficos observados no EMC associado ao disco são principalmente mudanças na forma do espaço do disco intervertebral e estenose do canal vertebral. Para melhor diagnóstico, seria necessário uma mielografia ou tomografia computadorizada (TC), porém não era viável para o tutor no momento.

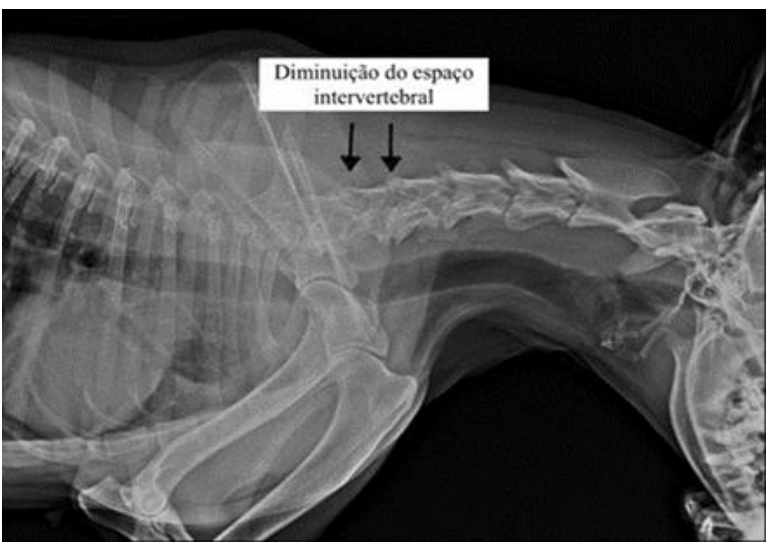

Figura 1. Radiografia Cervical aterolateral direita

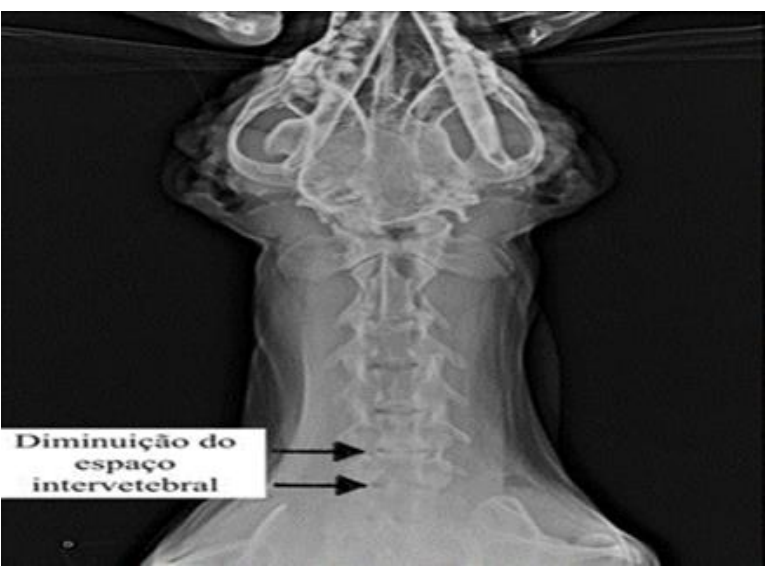

Figura 2. Radiografia Cervical ventrodorsal

\section{Resultados e Discussão}

Costa (2010) diz que avaliar a marcha do paciente é o componente mais importante do exame em cães suspeito de ter espondilomielopatia cervical, porque identifica de forma confiável ataxia proprioceptiva, mesmo na ausência de déficits proprioceptivos conscientes, sendo assim, no dia 1 do tratamento fisioterapêutico, foi realizada uma avaliação física, onde foi observada mobilidade reduzida dos quatro membros, sendo mais perceptível nos membros torácicos, onde o animal apresentava maior rigidez ao andar, ao contrário do que diz Costa (2010) que relata que os cães afetados tipicamente têm ataxia mais evidente nos membros pélvicos e com anormalidades mais leves nos membros torácicos. A amplitude dos membros apresentava-se normal e a propriocepção dos quatro membros se apresentava normal.

Foram realizadas 2 a 3 sessões (dependendo da disponibilidade do tutor) semanais de fisioterapia. Nas suas sessões foi realizada a mobilização passiva de todas as articulações, que segundo Levine et al. (2008), estes exercícios são indicados no tratamento da dor e na perda de mobilidade secundária à disfunção neuromúsculo-esquelética. Os exercícios foram iniciados pela articulação coxofemoral, realizando movimentos circulares $\left(360^{\circ}\right)$; posteriormente, a articulação fêmorotíbio-patelar, com movimentos passivos de extensão e flexão; depois a articulação társica com movimentos de flexão, extensão e laterais; logo após, foram realizados movimento de extensão e flexão na articulação metatarsofalangeana. Depois dos membros posteriores, foram realizados movimentos passivos nos membros anteriores, movimentando as articulações escápulo-umeral, úmero-rádio-ulnar, cárpica e metacarpofalangeana com movimentos de extensão e flexão. Todos as mobilizações com 30 repetições, em média. A mobilização cervical foi realizada com a finalidade de aumentar a flexibilidade, para evitar a formação de aderências entre os tecidos moles e ossos, fibrose periarticular, para remodelar e melhorar a extensibilidade do músculo e outros tecidos, para ajudar e evitar o agravamento das lesões, como já citado por Millis et al. (2004), esses exercícios foram realizados com muita cautela, apenas sendo feito o movimento de flexão, pois na extensão, o animal indicava dor. Posteriormente às mobilizações, foram realizados alongamentos dos membros torácicos e pélvicos, por um minuto em cada grupo muscular, que segundo Millis et al. (2004) é importante para evitar o agravamento das lesões sejam elas nas articulações, músculos, tendões e/ou ligamentos. Após os alongamentos e mobilizações, o animal foi posicionado na bola suíça, que segundo Craig (2005), tem a finalidade de alongar, fortalecer a musculatura, reeducação da postura e treino de equilíbrio. 
Após todos os exercícios foi aplicado o TENS, que é uma técnica que aplica níveis baixos de corrente elétrica, que atua aumentando a amplitude dos movimentos e da força muscular melhorando a funcionalidade, diminuição da atrofia, analgesia, diminuição do edema e do espasmo muscular e alívio de contraturas, como citado por Levine et al. (2008). Com um eletrodo na altura da vértebra $\mathrm{C} 5$ e o outro na altura da $\mathrm{C} 7$. O modo do TENS escolhido foi o modo BURST, pela paciente apresentar dor crônica, com $200 \mu \mathrm{s}$ de largura de pulso e os outros parâmetros já estabelecidos pelo aparelho (Neurodyn ${ }^{\circledR}$ portátil), como a frequência em $2 \mathrm{~Hz}$, por 30 minutos. Juntamente com o TENS, foi utilizado uma bola com cravos para realizar uma massagem relaxante no animal, que segundo (Macías (1998); Dunning et al. (2005); Saunders (2007), a massagem manual, ou seja, sem uso de equipamentos, tem indicação para os casos em que se deseja diminuir a dor, promover o relaxamento, melhorar a amplitude de movimentos e a circulação sanguínea.

No dia 5 da fisioterapia o cão começou a andar com menos dificuldades nos membros posteriores, apresentando amplitude perto na normalidade. A tutora relatou que o animal começou a ter mais força nos membros para se manter em pé na hora de realizar micção e defecar. No dia 7 da fisioterapia, o animal se levanta com menor dificuldade e em menos tempo. Também foi observado que sua pupila se apresentava em tamanho perto na normalidade, o que pode ser um indicativo de alívio da dor. A partir do dia 07 da fisioterapia, foi complementado ao tratamento fisioterapêutico, a administração, por uso oral, de Cloridato de Tramadol na dosagem de $2.5 \mathrm{mg} / \mathrm{kg}$ a cada $12 \mathrm{~h}$, como analgésico e, para maior analgesia, foi combinado com Dipirona $2.5 \mathrm{mg} / \mathrm{kg}$ a cada 12 horas.

Após 15 sessões de fisioterapia, o animal apresentava melhoras significativas, no qual já conseguia fazer todas suas atividades cotidianas como deitar e levantar, defecar e urinar sem perder o equilíbrio, aumentou a amplitude de movimento da cervical e dos MMPP. Os MMTT ainda se apresentavam rígidos ao andar. Como foi citado por Costa (2010), a decisão de recomendar o tratamento cirúrgico deve basear-se na gravidade dos sinais neurológicos, o grau de dor e tipo de gravidade das lesões compressivas. Apesar do animal ter apresentado melhoras significativas, a compressão medular não seria diminuída e havia também uma possível calcificação entre vertebras, que pôde ser confirmada através de uma TC que foi realizada após todas as sessões de fisioterapia, confirmando as suspeitas de Síndrome de Wobbler.

$\mathrm{Na}$ TC, tivemos como alguns resultados: material extraído localizado centralmente no canal medular, com aspecto pouco mineralizado (aproximadamente 85 unidades Hounsfield), com dimensões aproximadas de $1,27 \mathrm{~cm}$, estendendo cranialmente $0,58 \mathrm{~cm}$ e caudalmente $0,69 \mathrm{~cm}$, promovendo compressão importante de canal medular e forames intervertebrais; diminuição dos espaços intervertebrais C4-C5, C5-C6 e C7-T1, sendo mais severo em C5-C6, com presença de importante esclerose de osso sub-condral e presença de proliferação osteofítica em ponte entre C5-C6. O animal foi encaminhado de volta ao neurologista, para que com a clínica atual juntamente com os resultados tomográficos, seja decidido se o cão será encaminhado para a cirurgia ou não.

\section{Conclusão}

Apesar do animal ter sido encaminhado para o neurologista para reavaliação e possível encaminhamento cirúrgico, a melhora no quadro clínico de dor, da mobilidade dos membros, amplitude de movimento e melhora nas atividades cotidianas do animal foram eficientes principalmente pelas observações da tutora. $\mathrm{O}$ tratamento fisioterapêutico terá continuidade indo o animal para a cirurgia ou não. A fisioterapia veterinária vem sendo uma alternativa muito procurada pelos tutores por apresentar uma conduta terapêutica não invasiva, onde o tutor pode acompanhar o progresso do animal de perto e participar do tratamento.

\section{Referências Bibliográficas}

Costa R.C. 2010. Cervical spondylomyelopathy (wobbler syndrome) in dogs. Veterinary Clinics: Small Animal Practice 40, 881-913.

Craig C. 2005. Pilates com a bola. Phorte, São Paulo, Brasil.

Dunning D., Halling K.B. \& Ehrhart N. 2005. Rehabilitation of medical and acute care patients. Veterinary Clinics: Small Animal Practice 35, 1411-26.

Levine D., Millis D.L., Marcellin-Little D.J. \& Taylor R. 2008. Reabilitação e fisioterapia na prática de pequenos animais. Roca, São Paulo, Brasil. 
Macías M.T.D. 1998. Massoterapia. In: Manual de Medicina Física (eds. by Morillo MM, Vega JMP \& Portero FS). Harcourt Brace, Madrid, Spain.

Millis D.L., Levine D. \& R.A. T. 2004. Range-ofmotion and stretching exercises. In: Canine rehabilitation and physical therapy (eds. by Millis DL, Levine D \& Taylor RA). Saunders, Philadelphia, USA.

Saunders W.B. 2007. Kirk's Current Veterinary Therapy, Philadelphia.
Steiss J.E. \& Levine D. 2005. Physical agent modalities. Veterinary Clinics: Small Animal Practice 35, 1317-33.

Recebido: 15 Mai. 2018.

Aprovado: 5 Jun. 2018

Publicado: 14 Jul. 2018

Licenciamento: Este artigo é publicado na modalidade Acesso Aberto sob a licença Creative Commons Atribuição 4.0 (CC-BY 4.0), a qual permite uso irrestrito, distribuição, reprodução em qualquer meio, desde que o autor e a fonte sejam devidamente creditados. 